\title{
Hereditary breast cancer: screening and risk reducing surgery
}

\author{
Matteo Renzulli ${ }^{1 \#}$, Simone Zanotti ${ }^{2 \#}$, Alfredo Clemente ${ }^{3}$, Giangaspare Mineo ${ }^{4}$, Francesco Tovoli ${ }^{5}$, \\ Alfonso Reginelli ${ }^{3}$, Antonio Barile ${ }^{6}$, Salvatore Cappabianca ${ }^{3}$, Mario Taffurelli ${ }^{2 *}$, Rita Golfieri ${ }^{1 *}$ \\ ${ }^{1}$ Radiology Unit, Department of Experimental, Diagnostic and Speciality Medicine, Sant'Orsola Hospital, University of Bologna, Bologna, Italy; \\ ${ }^{2}$ Breast Unit, Department of Woman, Child and Urological Diseases, Sant'Orsola Hospital, University of Bologna, Bologna, Italy; ${ }^{3}$ Radiology \\ and Radiotherapy Unit, Department of Precision Medicine, University of Campania "L. Vanvitelli", Naples, Italy; ${ }^{4}$ Radiology Unit, Department \\ of Diagnostic Medicine and Prevention, Sant'Orsola Hospital, University of Bologna, Bologna, Italy; ${ }^{5}$ Unit of Internal Medicine, Department of \\ Medical and Surgical Sciences, University of Bologna, Bologna, Italy; ${ }^{6}$ Department of Biotechnology and Applied Clinical Sciences, University of \\ L'Aquila, S. Salvatore Hospital, L'Aquila, Italy \\ Contributions: (I) Conception and design: M Renzulli, S Zanotti, A Clemente, M Taffurelli, R Golfieri; (II) Administrative support: M Renzulli, S \\ Zanotti, A Clemente, G Mineo, F Tovoli, R Golfieri; (III) Provision of study materials or patients: M Renzulli, S Zanotti, G Mineo, M Taffurelli, R \\ Golfieri; (IV) Collection and assembly of data: M Renzulli, S Zanotti, A Clemente, F Tovoli, A Reginelli, A Barile, S Cappabianca, R Golfieri; (V) \\ Data analysis and interpretation: M Renzulli, A Clemente, G Mineo, A Reginelli, A Barile, M Taffurelli, R Golfieri; (VI) Manuscript writing: All \\ authors; (VII) Final approval of manuscript: All authors. \\ \#These authors contributed equally to this work. \\ *These authors equally contributed as joint senior author. \\ Correspondence to: Alfredo Clemente, MD. Radiology and Radiotherapy Unit, Department of Precision Medicine, University of Campania "L. \\ Vanvitelli”, Piazza Miraglia 2, 80138 Naples, Italy. Email: alf.clemente@hotmail.it.
}

Background: The screening modalities for women at high risk for breast cancer has received an increasing role during the last years. The aim of this study was to evaluate the performance of our screening program comparing the diagnostic sensitivity of clinical breast examination, mammography, ultrasonography (US) and magnetic resonance imaging (MRI).

Methods: Clinical Breast examination, mammography, US and MRI for each patient with BRCA1 and $B R C A 2$ mutation who underwent breast surgery in our Institution from October 2008 to April 2016 were retrospectively evaluated. The diagnostic accuracy for MRI and for the other surveillance tests in identifying early breast cancer were assessed.

Results: Twenty-six female patients with genetic mutation underwent breast surgery. Twenty-two out of 26 (85\%) developed cancer during the dedicated screening protocol whereas 4 women who underwent surgery did not have cancer. Imaging was able to detect cancer in all 22 patients (per patient sensibility of 100\%), identifying all 35 neoplastic lesions (per lesion sensibility of 100\%). The combination of Clinical Breast Examination, US and mammography aided the cancer diagnosis in 14 (64\%) of patients with a sensitivity of $64 \%$ and specificity of $100 \%$. MRI identified all the cancers, with sensibility and specificity of $100 \%$. Moreover, in 8 (36\%) of the 22 patients who developed breast cancers, the cancers were detected only by MRI, revealing a significant superiority respect to the other surveillance modalities $(\mathrm{P}<0.05)$.

Conclusions: MRI demonstrated to be the best imaging modality in detection of breast cancer even for lesion $<1 \mathrm{~cm}$. Prophylactic mastectomy is the most effective risk reduction strategy in women at high risk, contributing to the reduction of anxiety related to the condition of a carrier.

Keywords: Breast cancer; magnetic resonance imaging (MRI); mammography; ultrasound; screening

Submitted Jan 28, 2019. Accepted for publication Apr 04, 2019.

doi: 10.21037 /gs.2019.04.04

View this article at: http://dx.doi.org/10.21037/gs.2019.04.04 


\section{Introduction}

Breast cancer is the most common cancer in in all women at any age (29\% of all female tumours) and is the first leading cause of cancer related death in women (1). It has a variable prevalence according to the geographical areas showing an increasing incidence rates in industrialized countries (1). However, the incidence rate has showed only a modest increase during the last years, thanks to the development of screening programs started from the 2010 predominantly in patients between 45-49 years of age (1). The mortality has had a decrease of $1.96 \%$ /year while the survival is attested at $89.7 \%$ at 5 years (2). There are various recognized risk factors for the development of breast cancer including: early menarche, late menopause, nulliparity, breastfeeding failure, age, physical activity, radiation, alcohol, obesity, estrogen replacement therapy (the incidence of breast cancer is greater than $30 \%$ if in estrogen-only therapy and is double if in estrogen-progestin therapy) $(3,4)$. However, the most important risk is the hereditary factor: the individual risk, in fact, increases directly proportional to the number of affected relatives and with the decrease of the age onset of the first carcinoma (5). Almost half of these cases (5\% of all breast cancers) are caused by hereditary forms with autosomal dominant transmission ( 2,500 cases/ year); women with these hereditary forms, have a risk of developing breast cancer during their lifetime of about $60-70 \%$ and develop tube-ovarian carcinoma of $20-40 \%$, thus being genetically predisposed for what is defined hereditary breast and ovarian cancer (HBOC) (6).

The genes that confer the highest risk of developing hereditary breast cancer ("high penetrance" genes) are the breast cancer 1 (BRCA1), located on the long arm of chromosome 17 and the breast cancer 2 (BRCA2), located on the long arm of the chromosome 13 (7). Some studies report a risk of developing $60-80 \%$ lifetime breast cancer in the presence of these mutations, which is much higher than the $12.5 \%$ risk of the general population $(2,8)$.

Breast cancer in women with BRCA mutations has particular characteristics: it occurs in early age, is often bilateral, multifocal and multicentric, and the risk of developing contralateral cancer is greater than $30 \%$ 10 years after the first event (9).

The relevance assumed by the hereditary risk of breast cancer has led to international public health programs aimed to identify and manage patients with high risk factors. These patients have the possibility to choose different strategies to reduce their risk of developing breast cancer, such as intensive clinical and imaging surveillance, lifestyle modifications, chemoprevention and prophylactic surgery (10).

Imaging surveillance is not a true risk reduction strategy but aims to identify the neoplasm in the earliest stage. In this setting, magnetic resonance imaging (MRI) is the imaging modalities with the best sensibility for high-risk patients, as demonstrated by several studies (11-15). In fact, an annual breast MRI has a sensitivity of $81 \%$, compared to $40 \%$ of mammography and $42 \%$ of ultrasound; in addition, the high sensitivity combined with the absence of radiation means that this imaging technique represents the best screening examination recommended by the European guidelines (16-18). Furthermore, the combination of the mammography and MRI has demonstrated a sensitivity close to $100 \%$ (19).

Surgical prophylaxis remains the most effective therapeutic strategy to reduce the risk of developing breast cancer. It has a significant psychological impact on young women reducing not only the risk of cancer but also the anxiety $(20,21)$.

The aim of this study was to evaluate the performance of our screening program of women at genetic-familial high risk of developing breast cancer, comparing the diagnostic sensitivity of clinical breast examination, mammography, ultrasonography (US) and MRI. Moreover, this study aimed to assess the quality of life of women after prophylactic surgery.

\section{Methods}

This retrospective study obtained the ethical approval by the institutional review board and the requirement written informed consent was obtained from all patients.

\section{Patient population}

Twenty-six female patients with genetic mutation underwent breast surgery from October 2008 to April 2016 at the Breast Unit of our Institution. All patients had a family history of breast and/or ovarian cancer in first degree relatives. The clinical information's were obtained from an informatic system in which all data including genetic testing, imaging information's and pathologic features were collected.

\section{Genetic testing}

Genetic counselling and genetic testing for identification 


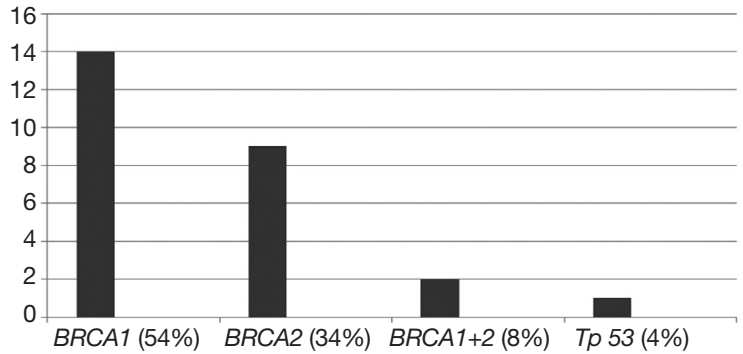

Figure 1 Graphical representation of breast cancer genes mutation in a sample of 26 patients.

of BRCA1 and BRCA2 mutations were performed including different molecular procedures in all patients. Only carriers with clearly pathogenic $B R C A 1$ and $B R C A 2$ mutations were included.

\section{Clinical and imaging analysis}

All patients with BRCA1 and BRCA2 mutation underwent dedicated screening protocol for high-risk women with no more than a 1-year interval between examinations (22). For each patient was performed a clinical breast examination, mammography, ultrasound (US) and contrast-enhanced MRI. All imaging examinations were retrospectively evaluated by three physicians with at least 10 years of experience in breast imaging. Clinical evaluation was performed in association with US examinations for all patients. Both breasts were systematically examined by using different US scans and clinical findings were correlated with imaging findings during reporting. Full field digital mammographic examination was performed with homogeneous breast compression and automatic exposure control in the bilateral standard, mediolateral oblique, and craniocaudal positions.

All MRI examinations were performed on a $1.5-\mathrm{T}$ superconducting system (Signa, GE Medical Systems, Milwaukee, WI, USA) using a dedicated breast coil. A gadolinium-based contrast agent (Gadoteridol, ProHance, Bracco, Milan, Italy) was administered to all patient at $2 \mathrm{cc} / \mathrm{sec}$ followed by a 20 -cc saline flush. Precontrast sequences included a 3-plane localizer, axial T2-weighted with fat saturation and diffusion weighted imaging ( $\mathrm{B}=0 ; \mathrm{B}=1,000)$ of each breast. Sagittal three-dimensional gradient echo T1-weighted dynamic imaging with and without chemical fat saturation, and eight times following contrast injection with approximately 70 seconds temporal resolution. Delayed high-resolution axial and sagittal T1- weighted fast spoiled gradient echo sequences with fat saturation were also obtained with the same sequence specifications.

\section{Surgical analysis}

All patients at high risk to develop a breast cancer underwent surgery. All surgical procedures were performed by two surgeons with almost 15 -year experienced. Different surgical techniques were performed, and different axillary nodes dissections were carried out according to the tumor stage and nodal involvement. Different breast reconstruction techniques were also performed after therapeutic mastectomy. The risk-reducing prophylactic mastectomies were performed after a surgical counselling, to patients with BRCA genes mutation who explicitly decided to undergo surgery to reduce their anxiety disorders.

\section{Statistical analysis}

Sensitivity, specificity, positive and negative predictive values and diagnostic accuracy were calculated for MRI alone or for the other surveillance tests alone or in combination.

The Chi-squared test was used to compare the sensitivities between the two sets of MRI alone and in combination with the other surveillance tests. A two-tailed $\mathrm{P}$ value of less than 0.05 was considered to indicate a statistically significant difference. All statistical analyses were performed using commercially available software (SPSS, version 11, SPSS, Chicago, IL, USA).

\section{Results}

Among the 26 patients with $B R C A 1$ and $B R C A 2$ mutations, only $4 \%$ developed the first neoplasm over the age of 50 , while $96 \%$ developed breast cancer below the age of 50 ( $57 \%$ between $30-45$ years of age) with an average age of 40.6 years (range, $24-56$ years).

\section{Type of mutations}

Fourteen out of the 26 patients examined (54\%) carried a mutation on the BRCA1 gene, $9(35 \%)$ a mutation on the $B R C A 2$ gene, $2(8 \%)$ had mutations on both $B R C A$ genes, and $4 \%(\mathrm{n}=1)$ a mutation on the $T p 53$ gene (Li Fraumeni syndrome). Fourteen out of 26 women examined (54\%) performed the genetic test after one or more surgical 
procedures, 5 (19\%) performed the genetic test after the first tumor diagnosis and 7 (27\%) performed the genetic test during the screening process performed due to the strong familiar risks (Figure 1).

\section{Personal bistory of carcinoma}

The $85 \%(n=22)$ of the patients had a personal history of breast cancer, for a total of 35 lesions, while 4 patients did not develop carcinoma; within the latter group, 1 patient presented a suspicious nodule that, after surgery, turned out to be a fibroadenoma while 3 patients (12\%) during the screening program decided to undergo bilateral prophylactic surgical intervention without cancer. The 22 women who developed breast cancer during the close clinical and imaging surveillance, underwent analysis of the axillary nodes to verify the presence of metastases. Ten out of 12 patients who underwent to sentinel lymph node biopsy, resulted negative for macro-metastasis $(>2 \mathrm{~mm})$ and 2 were positive for macro-metastasis $(>2 \mathrm{~mm}$ ). These two latter women underwent axillary nodes dissection up to the III level nodes. The remaining 10 patients underwent to axillary node dissection.

\section{Tumor characteristics}

The most frequent neoplasm was the invasive ductal carcinoma, encountered in 29 out of 35 cases $(83 \%)$. The histological grading of 25 lesions $(71 \%)$ of the neoplasms was of the G3 type, with poor differentiation. In the most of cases $(19 / 35 ; 54 \%)$ the pathological profile was a triple negative profile and in 30 out of 35 women $(86 \%)$ the Ki-67 proliferation index, was significantly high (>20\%) and for this reason, patients carried out an adjuvant treatment (chemotherapy and radiotherapy).

\section{Breast imaging}

Of 26 women who underwent surgery, 22 patients developed cancers during the dedicated screening protocol whereas 4 women who underwent surgery did not have cancer. Imaging was able to detect the cancer in all patients (per patient sensibility of $100 \%$ ), identifying all 35 neoplastic lesions (per lesion sensibility of $100 \%$ ). The $57 \%$ of the lesion (20 out of 35) were between 1 and $2 \mathrm{~cm}$ while $25.7 \%$ (9 out 35) were above $2 \mathrm{~cm}$ and $17.1 \%$ (6 out of 35 ) below $1 \mathrm{~cm}$ (range, $0.7-0.9 \mathrm{~cm}$ ). Only 12 of the 35 cancers were palpable. Eleven $(50 \%)$ of 22 patients had multifocal, multicentric or bilateral breast cancers. The combination of clinical breast examination, US and mammography aided the cancer diagnosis in 14 (64\%) of patients with a sensitivity of $64 \%$, specificity of $100 \%$, PPV of $100 \%$, NPV of $33 \%$ and diagnostic accuracy in $69 \%$. MRI identified all the cancers, with sensibility, specificity, PPV, NPV and diagnostic accuracy of $100 \%$. Moreover, in $8(36 \%)$ of the 22 patients who developed breast cancers, the cancers were detected only by MRI, therefore with statistically significant superiority as respect to the other surveillance modalities $(\mathrm{P}<0.05)$ (Figure 2).

\section{Surgical assessment}

Eleven out of 26 patients initially performed conservative surgery. Within 5 years, 8 out of 11 (73\%) developed recurrence that required a subsequent mastectomy with contralateral prophylactic mastectomy. Five out of 26 patients (19\%) underwent unilateral mastectomy as appropriate treatment without subsequent recurrence; all of them opted for a contralateral prophylactic mastectomy. A therapeutic mastectomy and prophylactic mastectomy in the same surgical time was performed in 5 patients without subsequent diagnosis of recurrence.

Four patients performed bilateral risk-reducing mastectomy without having a breast cancer. Only one patient performed bilateral therapeutic mastectomy for bilateral breast cancers.

\section{Risk-reducing surgery}

Twenty-three patients out of 26 with BRCA1 and BRCA2 deleterious mutations performed at least one risk-reducing surgery. A total of 46 mastectomies were performed while 26 out 46 were risk-reducing mastectomies: 3 patients with bilateral in healthy women without breast cancer; 1 patient with bilateral, in patient with a previous personal history of bilateral carcinoma; 18 patients with unilateral, in patients who had already performed or performed simultaneous a therapeutic mastectomy.

The surgical techniques used for the 26 prophylactic mastectomies were: 9 out of 26 (35\%) simple mastectomies; $16(62 \%)$ nipple sparing mastectomies; 1 (4\%) skin sparing mastectomy.

\section{Discussion}

All patients examined had one (16\%) or more (84\%) first- 

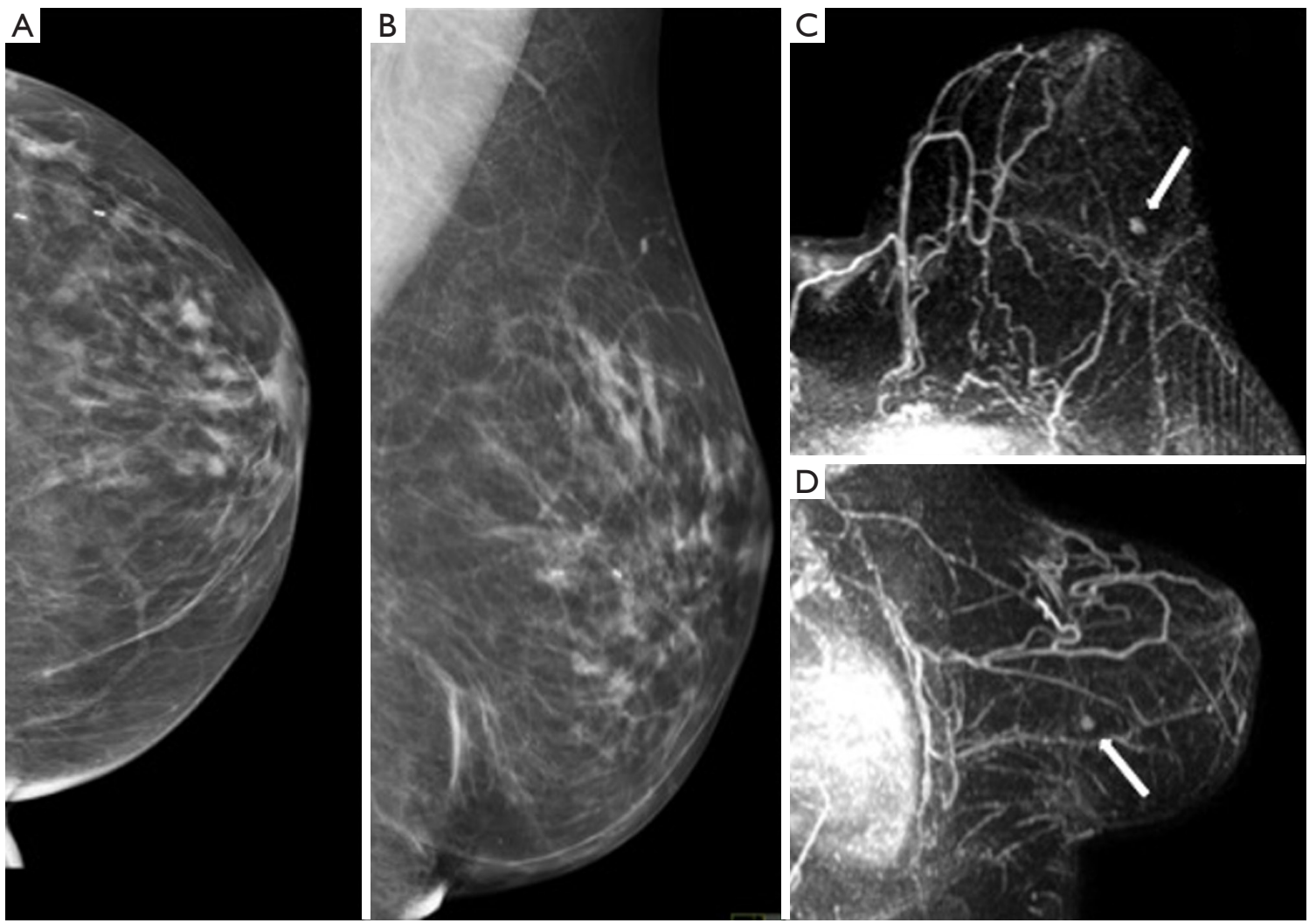

Figure 2 Asymptomatic 52-year-old woman with BRCA1 mutation. (A) Craniocaudal and (B) mediolateral oblique mammograms of the left breast did not detect any suspicious abnormality. Maximum intensity projection reconstructions of axial (C) and sagittal (D) contrast enhanced T1-weighted gradient-echo breast MRI showed an enhancing 4 mm-mass (arrows) in the lateral quadrant of the left breast only 1 week after mammographic examine.

degree relatives suffering from breast and/or ovarian cancer; only $27 \%(\mathrm{n}=7)$ were already aware of their $B R C A 1$ or $B R C A 2$ deleterious mutations, while $73 \%(\mathrm{n}=19)$ performed the genetic test only after the diagnosis of breast cancer or surgical intervention. This is a very important feature because it highlights the number of women affected by $B R C A 1$ or BRCA2 mutations (high penetrance genes for breast cancer) which is still underestimated. It is interesting that among the 26 patients examined, only $6(23 \%)$ underwent surgery from October 2009 to December 2013 (4 years), while the remaining 20 (77\%) performed the riskreducing intervention from January 2014 to April 2016 ( 2 years). This emphasizes the crescent interesting during the last years about this issue. The $96 \%$ among surveilled patients, developed breast cancer under 50 years: in particular $60 \%$ between $30-45$ years (mean age 40.7 years). This data agrees with the literature, in fact patients with genetic mutation for breast cancer develop neoplasm at an earlier age than sporadic forms (2,6-9).

Analyzing the tumour characteristics arisen in patients with BRCA1 and BRCA2 mutations, the most frequent features were: invasive ductal carcinoma ( $83 \%)$; high histological grade G3 (72\%); triple negative carcinomas (54\%); greater proliferative index $(\mathrm{Ki}-67$ index $>86 \%$ of patients); increased risk of ipsilateral and/or contralateral carcinoma. These findings are in line with those reported in literature, suggesting an aggressive clinical course of these tumors $(3,8,12,23,24)$. The screening examinations and the early diagnosis of breast cancer in these group of patients, has received a considerable importance during the last years. It is well known that contrast enhanced MRI have higher sensitivity for cancer detection in women at high risk than others imaging modalities (11,19,25-27). In our population MRI examination has demonstrated the high sensitivity (100\%) in detecting neoplastic lesions, including in those lesions $<1 \mathrm{~cm}$. Our results are in line 
with many studies that reported a sensitivity of breast MRI between 86-97\% with a high diagnostic accuracy of the early stages $(19,25-31)$. However, the combination of clinical breast examination, mammography and US has not sufficiently sensitive in high-risk women, as demonstrated by many series $(11,25)$ and consequently, has a minor role in patients with hereditary predisposition to the disease. In fact, this diagnostic combination has demonstrated a sensitivity of $64 \%$ in our BRCA mutation carriers and a lower sensitivity than its estimated (83\%) for detection in the general population, as well known from many studies $(12,16,25,32,33)$. Furthermore, the higher sensitivity of MRI in lesion detection is consistent if considering its high capability to detect potential axillary nodal involvement. Our experience supports the high sensitivity of MRI in imaging detection of early breast cancers in women at high risk.

The surgical management of high-risk patients suggests different considerations. In our study population, 24 out of 26 patients underwent bilateral mastectomy. However, the awareness of mutation has significantly influenced both the therapeutic approach and the time between the first diagnosis of cancer and the prophylactic mastectomy. In fact, 11 patients who were already aware of being a carrier of BRCA mutation, decided to undergo a bilateral mastectomy with immediate reconstruction at the same time, while for the remaining 13 patients who did not know their condition of mutation carriers, the time between the first diagnosis and the prophylactic surgery was on average 6 years. Besides, the interval time between the detection of BRCA mutation and the risk-reducing surgery was on average 3 months. This emphasizes the importance of clinical counseling and the information received about the personal risk of developing cancer, the alternative strategies of prophylactic surgery and the awareness of the patient's benefit risk.

The risk-reducing technique, adopted in over $60 \%$ of the women, was the nipple sparing mastectomy, which is the most recent mastectomy technique offering the best aesthetic result and improves the post-intervention "quality of life" keeping the oncological radicality, as also confirmed by many authors (34-36).

However, our retrospective study has some limitations such as the small sample involved and the short followup time. Instead, the hereditary breast cancer screening programs are still developing and the risk-reducing surgical approach, also emphasized by the recent "Angelina Jolie" effect, is still the subject of extensive international studies based on health and mental implications.

\section{Conclusions}

In conclusion, our study confirms the importance of identifying women with mutation of high-penetrance genes for breast cancer, in order to assess their cancer risk and develop dedicated screening program with a certain level of intensity and involve patients in the most appropriate surgical strategy. In particular, in patient with mutation of high-penetrance genes for breast cancer, MRI demonstrated to be the best imaging modality in detection of breast cancer even for lesion $<1 \mathrm{~cm}$. Prophylactic mastectomy is the most effective risk reduction strategy, contributing to the reduction of anxiety related to the condition of a carrier, as well as obtaining good aesthetic results thanks to new techniques of conservative mastectomy and immediate breast reconstruction.

\section{Acknowledgments}

None.

\section{Footnote}

Conflicts of Interest: The authors have no conflicts of interest to declare.

Ethical Statement: The study was approved by the ethical approval by the institutional review board and written informed consent was obtained from all patients.

\section{References}

1. Ferlay J, Steliarova-Foucher E, Lortet-Tieulent J, et al. Cancer incidence and mortality patterns in Europe: estimates for 40 countries in 2012. Eur J Cancer 2013;49:1374-403.

2. Siegel RL, Miller KD, Jemal A. Cancer statistics, 2016. CA Cancer J Clin 2016;66:7-30.

3. Yang XR, Chang-Claude J, Goode EL, et al. Associations of breast cancer risk factors with tumor subtypes: a pooled analysis from the breast cancer association consortium studies. J Natl Cancer Inst 2011;103:250-63.

4. Beral V. Breast cancer and hormone-replacement therapy in the Million Women Study. Lancet 2003;362:419-27.

5. Lalloo F, Evans DG. Familiar breast cancer. Clinical Genetics 2012;82:105-14.

6. Singer CF, Tea MK, Pristauz G, et al. Clinical Practice Guideline for the prevention and early detection of breast 
and ovarian cancer in women from HBOC families. Wien Klin Wochenschr 2015;127:981-6.

7. Melchor L, Benìtez J. The complex genetic landscape of familiar breast cancer. Human Genetics 2013;132:845-63.

8. Chen S, Parmigiani G. Meta-analysis of BRCA1 and BRCA2 penetrance. J Clin Oncol 2007;25:1329-33.

9. Graeser MK, Engel C, Rhiem K, et al. Contralateral breast cancer risk in BRCA1 and BRCA2 mutation carriers. J Clin Oncol 2009;27:5887-92.

10. Paradiso A, Formenti S. Hereditary breast cancer: clinical features and risk reduction strategies. Ann Oncol 2011;22 Suppl 1:31-6.

11. Sardanelli F, Podo F. Breast MR imaging in women at high-risk of breast cancer. Is something changing in early breast cancer detection?. Eur Radiol 2007;17:873-87.

12. Veltman J, Mann R, Kok T, et al. Breast tumor characteristics of BRCA1 and BRCA2 gene mutation carriers on MRI. Eur Radiol 2008;18:931-8.

13. Kuhl CK, Schmutzler RK, Leutner CC, et al. Breast MR imaging screening in 192 women proved or suspected to be carriers of a breast cancer susceptibility gene: Preliminary results. Radiology 2000;215:267-79.

14. Tilanus-Linthorst MM, Obdeijn IMM, Bartels KC, et al. First experiences in screening women at high risk for breast cancer with MR imaging. Breast Cancer Res Treat 2000;63:53-60.

15. Reginelli A, Clemente A, Cardone C, et al. Computed tomography densitometric study of anti-angiogenic effect of regorafenib in colorectal cancer liver metastasis. Future Oncol 2018;14:2905-13.

16. Warner E, Plewes DB, Hill KA, et al. Surveillance of BRCA1 and BRCA2 mutation carriers with magnetic resonance imaging, ultrasound, mammography, and clinical breast examination. JAMA 2004;292:1317-25.

17. Lehman CD, Blume JD, Weatherall P, et al. Screening women at high risk for breast cancer with mammography and magnetic resonance imaging. Cancer 2005;103:1898-905.

18. Kriege M, Brekelmans CTM, Boetes C, et al. Efficacy of MRI and mammography for breast-cancer screening in women with a familial or genetic predisposition. $\mathrm{N}$ Engl J Med 2004;351:427-37.

19. Huzarski T, Górecka-Szyld B, Huzarska J, et al. Screening with magnetic resonance imaging, mammography and ultrasound in women at average and intermediate risk of breast cancer. Hered Cancer Clin Pract 2017;15:4.

20. Hartmann LC, Schaid DJ, Woods JE. Efficacy of bilateral prophylactic mastectomy in women with a family history of breast cancer. N Engl J Med 1999;340:77-84.

21. Rebbeck TR, Friebel T, Lynch HT. Bilateral prophylactic mastectomy reduces breast cancer risk in BRCA1 and BRCA2 mutation carriers: the PROSE Study Group. J Clin Oncol 2004;22:1055-62.

22. Paluch-Shimon S, Cardoso F, Sessa C, et al. Prevention and screening in BRCA mutation carriers and other breast/ ovarian hereditary cancer syndromes: ESMO Clinical Practice Guidelines for cancer prevention and screening. Ann Oncol 2016;27:v103-10.

23. Reginelli A, Calvanese M, Ravo V, et al. Management of breast cancer in elderly patients. Int J Surg 2014;12:S187-92.

24. Granito A, Marinelli S, Terzi E, et al. Metronomic capecitabine as second-line treatment in hepatocellular carcinoma after sorafenib failure. Dig Liver Dis 2015;47:518-22.

25. Lee MV, Katabathina VS, Bowerson ML, et al. BRCAassociated Cancers: Role of Imaging in Screening, Diagnosis, and Management. Radiographics 2017;37:1005-23.

26. Ciaglia GF, Martino A, Sayad K, et al. Management of Bone Metastases From Breast Cancer in Upper and Lower Body at the Same Time: A Case Report. World J Oncol 2016;7:57-8.

27. Sardanelli F, Podo F, D'Agnolo G, et al. Multicenter comparative multimodality surveillance of women at genetic-familial high risk for breast cancer (HIBCRIT study): interim results. Radiology 2007;242:698-715.

28. Piscaglia F, Iavarone $M$, Galassi $M$, et al. Cholangiocarcinoma in Cirrhosis: Value of Hepatocyte Specific Magnetic Resonance Imaging. Digestive Diseases 2015;33:735-44.

29. Di Grezia G, Somma F, Serra N, et al. Reducing Costs of Breast Examination: Ultrasound Performance and Inter-Observer Variability of Expert Radiologists Versus Residents. Cancer Invest 2016;34:355-60.

30. Palma BD, Guasco D, Pedrazzoni M, et al. Osteolytic lesions, cytogenetic features and bone marrow levels of cytokines and chemokines in multiple myeloma patients: Role of chemokine (C-C motif) ligand 20. Leukemia 2016;30:409-16.

31. Ferrari F, Arrigoni F, Miccoli A, et al. Effectiveness of Magnetic Resonance-guided Focused Ultrasound Surgery (MRgFUS) in the uterine adenomyosis treatment: technical approach and MRI evaluation. Radiol Med 2016;121:153-61. 
32. Reginelli A, Silvestro G, Fontanella G, et al. Validation of DWI in assessment of radiotreated bone metastases in elderly patients. Int J Surg 2016;33:S148-53.

33. Zoccali C, Rossi B, Zoccali G, et al. A new technique for biopsy of soft tissue neoplasms: a preliminary experience using MRI to evaluate bleeding. Minerva Med 2015;106:117-20.

34. King TA, Pilewskie M, Morrow M. Optimal surgical

Cite this article as: Renzulli M, Zanotti S, Clemente A, Mineo G, Tovoli F, Reginelli A, Barile A, Cappabianca S, Taffurelli M, Golfieri R. Hereditary breast cancer: screening and risk reducing surgery. Gland Surg 2019;8(Suppl 3):S142-S149. doi: 10.21037/gs.2019.04.04 management for high-risk populations. Breast 2015;24 Suppl 2:S91-5.

35. Rhiem K, SchmutzlerR. Impact of Prophylactic Mastectomy in BRCA1/2 Mutation Carriers. Breast Care 2014;9:385-9.

36. Rossi C, Mingozzi M, Curcio A, et al. Nipple areola complex sparing mastectomy. Gland Surg 2015;4:528-40. 